\title{
Effects of percentage reinforcement on choice in a concurrent chain schedule
}

JOHN W. SCHNEIDER, DEPARTMENT OF PSYCHOLOGY, HAR VARD UNIVERSITY, Cambridge, Mass. 02138

The effects of random reinforcement omissions (percentage reinforcement) on choice behavior was studied with three pigeons trained on a two-link concurrent chain schedule. A terminal-link $V I$ schedule on one key always produced primary reinforcement; a terminal-link VI-percentage reinforcement schedule on the other key produced with equal probability either reinforcement or blackout. Relative response rates in the choice links matched the relative rates of reinforcement associated with the terminal-link schedules when these were VI $30 \mathrm{sec}$ vs VI $30 \mathrm{sec}$ (50\%); VI $1 \mathrm{~min}$ vs VI $30 \mathrm{sec}$ (50\%); and VI $30 \mathrm{sec}$ vs VI $30 \mathrm{sec}$ (baseline). These results suggest that percentage reinforcement affects choice solely through its effect on rate of primary reinforcement.

Using concurrent chain procedures to study steady-state choice behavior, Autor (1960) and Herrnstein (1964) have found that relative rates of initial-link (choice) responses match the relative rates of reinforcement associated with the terminal links of each chain. This relation has been established for cases in which the terminal-link schedules of reinforcement are both VI, both VR, or VI and VR. Results from the latter comparison (Herrnstein, 1964) provide strong evidence that rate of reinforcement, measured as $\mathrm{rft} / \mathrm{h}$ rather than $\mathrm{rft} / \mathrm{response}$, is the critical variable determining the obtained matching relation.

The present experiment tests choice under conditions in which reinforcements are delivered in one terminal link according to a VI schedule, and in the other according to a VI-percentage reinforcement schedule. A percentage reinforcement (PR) schedule is defined (Ferster \& Skinner, 1957) as any given schedule on which a certain percentage of reinforcements are replaced, usually in random order, by some other schedule outcome (e.g., blackout). By altering the proportion of schedule completions that produce reinforcement, the rate of reinforcement on a given schedule may be varied without changing the programmed relation between responding and the production of schedule outcomes. In the concurrent chain procedure of the present experiment, comparisons between VI schedules with and without percentage reinforcement make it possible to separately evaluate the effects on choice of rate of primary reinforcement and rate of schedule outcomes as such.

Method

Three adult, male, White Carneaux pigeons were maintained at $80 \%$ of their free-feeding body weights while serving as Ss. All birds had prior experimental histories on a variety of procedures.

The experimental chamber contained two translucent response keys each operated by pecks of at least $10 \mathrm{~g}$ force. Each effective response to a lighted key produced an audible feedback click. Chamber illumination was provided by a $7-\mathrm{W}$ bulb (houselight), and white masking noise was continuously present in the chamber.

Birds were run in the same order on each of four conditions until performances were stable, requiring from 25 to 35 sessions per condition. In all conditions a concurrent chain procedure was employed having identically constructed but independent VI 1 min schedules in the initial links, and VI schedules (with or without PR) in the second (terminal) links. The sets of 12 intervals used to construct the various VI schedules were selected according to a constant probability progression described by Fleshler \& Hoffman (1962). In all conditions the concurrent first-link schedules were associated with white keylights; the terminal-link schedules were associated with green on the left key (LK) and red on the right key (RK). Each schedule outcome, produced by completion of a terminal-link schedule, consisted of either a 3 sec grain reinforcement or a $3 \mathrm{sec}$ blackout during which the chamber was completely darkened. During both reinforcements and blackouts the response keys were darkened and inoperative. Each reinforcement or blackout was followed by an immediate return to the initial links, and sessions ended when the obtained number of reinforcements and blackouts (if any) totalled 50 .

In Condition 1 a baseline performance was established by using VI $30 \mathrm{sec}$ schedules (no PR) in each of the terminal links. Condition 2 was identical except that completions of the terminal-link schedule on RK produced with equal probability either reinforcement or blackout; i.e., the terminal-link schedule on RK was changed from VI $30 \mathrm{sec}$ to VI $30 \mathrm{sec}(50 \%)$. A 32-state stepping switch scheduled reinforcements and blackouts in a pseudo-random sequence constrained so that no more than three outcomes of the same type occurred sequentially on RK.

Baseline was redetermined in Condition 3 by running birds on the same procedure employed in Condition 1. In Condition 4, the terminal-link schedule on LK was changed to VI $1 \mathrm{~min}$, and the VI $30 \sec (50 \%)$ schedule used in Condition 2 was again programmed for the terminal link on RK.

Results

Table 1 presents data averaged across Ss for the final five sessions of each condition. Relative rate of responding to RK. in the initial links was calculated as the number of initial-link responses to RK divided by the total number of initial-link responses during each session. Rates of reinforcement used to obtain the relative rates of reinforcement shown in the table were measured as the number of primary reinforcements delivered for responding on a key divided by the total time spent in the terminal link on that key. Durations of reinforcements and blackouts were excluded from all calculations.

As shown in Table 1, overall rate of responding to the two keys in the initial links was about the same in all conditions. In each condition, the relative initial-link response rate on a key approximately equaled the obtained relative rate of reinforcement in the terminal link. In no condition did the two measures differ by more than 0.05 , and individual performances were similar enough not to require separate consideration.

Relative terminal-link response rates varied only slightly across conditions (Table 1). For each bird the relative rate on RK was lowest in Condition 2, and slightly lower than the bird's mean baseline value in Condition 4 , although the magnitudes of the differences across conditions were small in all birds.

Table 1

Summary of Results.

Each cell entry is an average across Ss of values from the last five sessions of the condition. The terminal-link schedules of each condition are specified in the column headings with all values in seconds.

\begin{tabular}{cccccc} 
& \multicolumn{5}{c}{ Condition } \\
\cline { 3 - 6 } & LK: & VI 30 & VI 30 & VI 30 & VI 60 \\
Item & RK: & VI 30 & VI 30(50\%) & VI 30 & VI 30 (50\%) \\
\hline
\end{tabular}

Overall response rate in initial links (resp/min) 56.2 58.6 58.9

Relative response rate on RK, initial links

Relative rate of reinforcement on RK, terminal links

Relative response rate on RK, terminal links

0.55

0.50

0.54 


\section{Discussion}

The results of this experiment suggest that the effects of percentage reinforcement on choice in concurrent chain schedules may be accounted for exclusively in terms of its effects on rate of primary reinforcement. The comparisons made in Conditions 2 and 4 show that a terminal-link VI $30 \mathrm{sec}(50 \%)$ schedule is equivalent in its effects on choice to a VI $1 \mathrm{~min}$ schedule, as would be expected on the basis of rates of reinforcement. Thus it appears that the matching relation between relative choice and relative rate of reinforcement found by Herrnstein (1964) may be extended to cases involving terminal-link PR schedules. It is clear, however, that a wider range of $P R$ schedules must be explored to confirm the generality of this conclusion.

It is of interest to note that the blackouts delivered in the PR conditions appeared, in themselves, to have no effect on choice. Single-key studies involving PR contingencies have typically found that rates and patterns of responding in PR schedule components remained similar to those obtained when all components terminated with primary reinforcement (Chung \& Neuringer, 1967; Neuringer \& Chung, 1967). That this effect may be ascribed to possible reinforcing properties acquired by the blackout stimuli in such schedules appears unlikely from the results of the present experiment, since blackouts had no evident effect on choice above and beyond the effects of rate of primary reinforcement.

In agreement with results from single-key studies, decreasing rate of reinforcement through percentage reinforcement of schedule components had little effect on rate of responding in the nonconcurrent terminal schedules. In Condition 2, where rates of schedule outcomes were equal on the two keys, the rate of reinforcement on RK was half that of LK, yet the relative rate of responding on $\mathrm{RK}$ in the terminal links was only slightly lower than the baseline values obtained with equal rates of reinforcement. Terminal-link response rates also remained close to baseline values when rates of reinforcement were equal and rates of schedule outcomes were in a two-to-one ratio (Condition 4).

\section{REFERENCES}

AUTOR, S. M. The strength of conditioned reinforcers as a function of frequency and probability of reinforcement. Unpublished doctoral dissertation, Harvard Univ., 1960.

CHUNG, S. H., \& NEURINGER, A. J. Control of responding by a percentage reinforcement schedule. Psychon. Sci, 1967, 8, 25-26.

FERSTER, C. B., \& SKINNER, B. F. Schedules of reinforcement. New York: Appleton-Century-Crofts, 1957.

FLESHLER, M., \& HOFFMAN, H. S. A progression for generating variable-interval schedules. J. exp. Anal. Behav., 1962, 5, 529-530.

HERRNSTEIN, R. J. Secondary reinforcement and rate of primary reinforcement J. exp. Anal. Behav., 1964, 7, 27-36.

NEURINGER, A. J., \& CHUNG, S. H. Quasi-reinforcement: control of responding by a percentage-reinforcement schedule. J. exp. Anal. Behav., $1967,10,45-54$. 